\title{
The Effect of Nebulizer Position on Aerosolized Epoprostenol Delivery in an Adult Lung Model
}

\author{
Allison C Anderson MSc RRT CHSE, Meagan N Dubosky MSc RRT, Kelly A Fiorino MSc RRT, \\ Vanessa Quintana RRT, Carl A Kaplan MD, and David L Vines MHS RRT FAARC
}

\begin{abstract}
BACKGROUND: Aerosolized epoprostenol is an alternative for inhaled nitric oxide in the management of pulmonary arterial hypertension and possibly acute hypoxemia. Our objective was to determine differences in drug deposition based on different nebulizer positions in the ventilator circuit, using a vibrating mesh nebulizer. METHODS: An 8.0-mm inner diameter endotracheal tube (ETT) was connected to a training test lung, compliance of $30 \mathrm{~mL} / \mathrm{cm} \mathrm{H}_{2} \mathrm{O}$, with a collecting filter placed at the ETT-test lung junction. A mechanical ventilator, heated wire circuit, and pass-over humidifier were utilized. A syringe pump continuously instilled a $15,000-\mathrm{ng} / \mathrm{mL}$ epoprostenol solution at 30,50 , and $70 \mathrm{ng} / \mathrm{kg} / \mathrm{min}$ into the vibrating mesh nebulizer at all 4 positions. Tidal volumes $\left(V_{T}\right)$ were set at 4,6 , and $8 \mathrm{~mL} / \mathrm{kg}$ for a $70-\mathrm{kg}$ patient with breathing frequencies of 25,16 , and 12 breaths/min, respectively. Epoprostenol was eluted from the filters $($ no. $=180)$ and analyzed with ultraviolet-visible spectrophotometry at $205 \mathrm{~nm}$ to estimate drug deposition. RESULTS: Epoprostenol deposition increased significantly $(P=.02)$ as the dosage increased from $30 \mathrm{ng} / \mathrm{kg} / \mathrm{min}$ (median 4,520.0 ng, interquartile range [IQR] 2,285.0-6,712.2 $\mathrm{ng}$ ) to $50 \mathrm{ng} / \mathrm{kg} / \mathrm{min}$ (median 6,065.0 ng, IQR 3,220.0-13,002.5 ng) and 70 ng/kg/min (median 9,890.0 ng, IQR 6,270.0-16,140.0 ng). No significant difference was found between variations in ventilator settings. No difference in deposition was found between the humidifier inlet and outlet, but these positions resulted in greater deposition compared with the inspiratory limb and between the ETT and Y-piece. CONCLUSIONS: The greatest amount of mean epoprostenol deposition resulted with the nebulizer placed at the humidifier inlet or outlet in a ventilator with bias flow. Key words: epoprostenol; aerosol drug therapy; pulmonary hypertension; nebulizers; vasodilator drugs. [Respir Care 2017;62(11):1387-1395. ( 2017 Daedalus Enterprises]
\end{abstract}

\section{Introduction}

Selective pulmonary vasodilators can be utilized in the management of patients diagnosed with ARDS or pulmo-

\footnotetext{
Ms Anderson, Ms Dubosky, Dr Kaplan, and Mr Vines are affiliated with the Division of Respiratory Care, Department of Cardiopulmonary Sciences, Rush University, Chicago, Illinois. Ms Fiorino is affiliated with the University of Michigan Hospital, Ann Arbor, Michigan. Ms Quintana is affiliated with the Rush University Medical Center, Chicago, Illinois.

Ms Anderson presented a version of this work at the AARC Congress held November 7-10, 2015, in Tampa, Florida.

Mr Vines has disclosed relationships with Carefusion, Covidien, Bayer Healthcare, Boehringer Ingelheim, Teleflex, Aerogen, and Halyard. Ms Dubosky has disclosed relationships with Aerogen and Halyard. The other authors have disclosed no conflicts of interest.
}

nary arterial hypertension. Administration of selective pulmonary vasodilating agents directly to the pulmonary vasculature allows for improved oxygenation, whereas the risk of systemic hypotension is significantly minimized. ${ }^{1}$

Inhaled nitric oxide (INO) decreases pulmonary artery pressure and pulmonary vascular resistance through relaxation of the musculature surrounding the pulmonary vasculature. ${ }^{1-3}$ This occurs through the activation of cytosolic guanylate cyclase, which augments the concentration of cyclic guanosine $3^{\prime} 5^{\prime}$-monophosphate. ${ }^{1}$ This process pro-

\footnotetext{
Correspondence: Allison C Anderson MSc RRT CHSE, Rush University, Department of Cardiopulmonary Sciences, Chicago, IL 60212. E-mail: allison_c_anderson@rush.edu.
}

DOI: $10.4187 /$ respcare. 05344 
duces vasodilation throughout the pulmonary vasculature, resulting in reduced pulmonary artery pressure, pulmonary vascular resistance, and shunting, which can be associated with improved oxygenation and ventilation/perfusion

\section{See the Related Editorial on Page 1504}

matching. ${ }^{1-3}$ Despite these benefits, INO has not been shown to produce a consistent reduction in mortality or ventilator-dependent days. ${ }^{1,2}$ Additionally, the potential for toxicity-related adverse outcomes has become a concern with INO. ${ }^{4}$ The use of INO may be associated with methemoglobinemia and worsening pulmonary hypertension as therapy is withdrawn. ${ }^{5}$

Afshari et $\mathrm{al}^{3}$ conducted a systematic review and metaanalysis of 14 randomized controlled trials (1,303 subjects) involving the use of INO for ARDS and found that there was no statistically significant difference in mortality between INO and control groups. The use of INO also did not affect the number of days that mechanical ventilation was required or the length of ICU or hospital stay. ${ }^{3}$ The risk of renal failure was also increased, and although the exact mechanism of renal injury during INO therapy is unknown, mitochondrial damage is believed to play a role., ${ }^{2,3} \mathrm{Al}-$ though oxygenation is improved with INO administration, the authors noted that any improvement is temporary and is limited to the initial $24 \mathrm{~h}$ of therapy. ${ }^{3}$

Epoprostenol is a potential alternative to INO in patients with ARDS. Epoprostenol is the pharmacologic form of prostacyclin, a naturally occurring prostanoid. ${ }^{6,7}$ Together with prostacyclins, prostaglandins, leukotrienes, and lipoxins are classified as eicosanoids. These lipid-derived molecules influence various physiologic responses throughout the body, including vasoconstriction and vasodilation, platelet aggregation, bronchoconstriction and bronchodilation, gastrointestinal motility, uterine contraction, renal filtration and renal blood flow, and inflammation. ${ }^{1,7,8}$ Epoprostenol was initially introduced for intravenous use. However, the intravenous route of administration is not selective to the pulmonary vasculature and has the potential to cause systemic hypotension. ${ }^{2,8}$ Intravenous administration also necessitates the placement of a long-term central venous catheter and the use of an infusion pump, increasing the risk of infection. ${ }^{6,8}$

To date, no randomized controlled trials have shown improved outcomes with the use of aerosolized epoprostenol for patients diagnosed with ARDS. ${ }^{2}$ Smaller studies have shown an improved $\mathrm{P}_{\mathrm{aO}_{2}} / \mathrm{F}_{\mathrm{IO}_{2}}$ and shunting, at margins similar to INO. 5 Torbic et $\mathrm{al}^{5}$ conducted a retrospective cohort analysis of 105 subjects receiving mechanical ventilation. The diverse patient population was analyzed by diagnostic subgroups to control for differences related to specific pathologic processes and disease states. A sta-

\section{QUICK LOOK}

\section{Current knowledge}

Inhaled epoprostenol is used to facilitate pulmonary vasodilation in critically ill mechanically ventilated patients. Use is increasing because the treatment is cost-effective and may have fewer systemic adverse effects when compared with intravenous treatment with epoprostenol. Clinical data are lacking regarding the effect that nebulizer placement within the mechanical ventilation circuit has on drug deposition at various ventilator settings.

\section{What this paper contributes to our knowledge}

Our study demonstrates that the greatest epoprostenol deposition occurred when the nebulizer was placed at the humidifier inlet or outlet in a ventilator with bias flow. We also found higher deposition as tidal volumes and dose increased.

tistically significant difference was not found in the $\mathrm{P}_{\mathrm{aO}_{2}} / \mathrm{F}_{\mathrm{IO}_{2}}$ between the INO and aerosolized epoprostenol groups, regardless of diagnosis or disease state. ${ }^{5}$ Despite the lack of evidence supporting the use of aerosolized epoprostenol in ARDS, the appeal is mainly associated with costs. The expense of INO ranges from 4.5 to 17 times more than aerosolized epoprostenol.,2,5

Variation in dosing practices and nebulization equipment utilized to administer aerosolized epoprostenol necessitates larger studies to determine precise dosing protocols and accurate and consistent delivery systems. In the current literature, protocols have initiated aerosolized epoprostenol at $0.05 \mu \mathrm{g} / \mathrm{kg} / \mathrm{min}(50 \mathrm{ng} / \mathrm{kg} / \mathrm{min})$ and reduced the dose by $0.01 \mu \mathrm{g} / \mathrm{kg} / \mathrm{min}(10 \mathrm{ng} / \mathrm{kg} / \mathrm{min})$ every 1-2 h until discontinuation. ${ }^{5}$ Other trials have initiated lowdose aerosolized epoprostenol ( $1-5 \mathrm{ng} / \mathrm{kg} / \mathrm{min})$ and titrated upward. ${ }^{2}$ The objective of this study was to evaluate the optimal delivery setup using a vibrating mesh nebulizer at different dosages and tidal volumes $\left(\mathrm{V}_{\mathrm{T}}\right)$.

\section{Methods}

A bench study was conducted in the aerosol laboratory at a large, urban, academic tertiary care medical center in Chicago, Illinois.

\section{Lung Model and System Design}

All trials of the study were run with an 8.0-mm inner diameter endotracheal tube connected to a 2-chambered training test lung (Michigan Instruments, Grand Rapids, Michigan) (Fig. 1). The distal end of the ETT was cut 


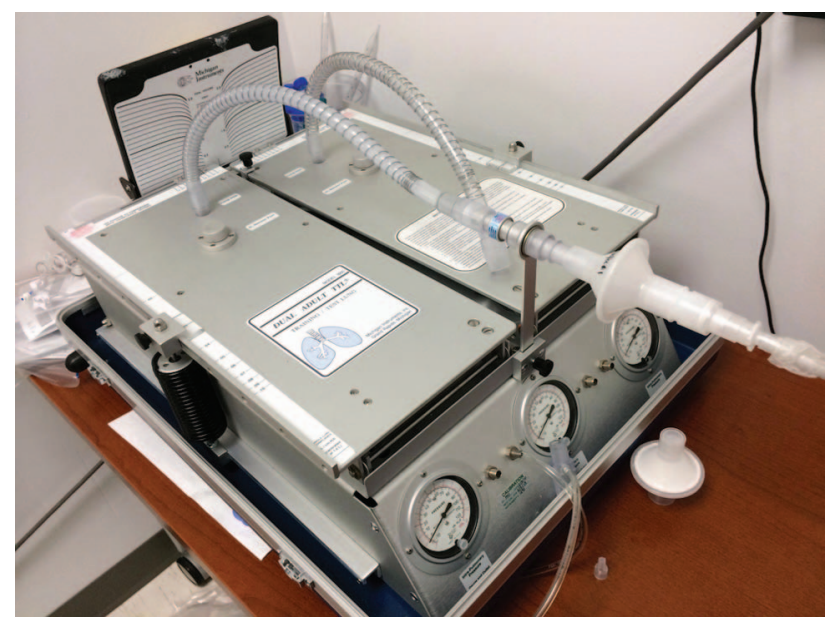

Fig. 1. Test lung, filter, and endotracheal tube.

immediately above the Murphy eye, perpendicular to the plane of the ETT, to allow a straight edge and tight fit between the distal end of the ETT and the adapter connecting it to the collecting filter (AirLife nonconductive respiratory therapy filter, bacterial/viral retentive, Carefusion, San Diego, California). The filter was then connected to the training test lung, the parameters of which were adjusted to reflect a resistance of $5 \mathrm{~cm} \mathrm{H}_{2} \mathrm{O} / \mathrm{L} / \mathrm{s}$ and a compliance of $30 \mathrm{~cm} \mathrm{H}_{2} \mathrm{O}$. The nebulizer was placed at the humidifier inlet, the humidifier outlet, within the inspiratory limb of the circuit, and between the endotracheal tube and Y-piece to compare drug deposition concentrations between nebulizer positions.

\section{Ventilator Settings}

The Puritan Bennett 840 ventilator (Covidien, Mansfield, Massachusetts) was used with a heated wire circuit (Carefusion) and an MR 850 pass-over humidifier (Fisher \& Paykel, Auckland, New Zealand), with the heater set to $37^{\circ} \mathrm{C}$ for invasive ventilation (Fig. 2). A short self test was run on the ventilator before beginning experimental runs.

Ventilator settings were determined based on a $70-\mathrm{kg}$ ideal body weight patient model. To deliver a consistent minute volume of $100 \mathrm{~mL} / \mathrm{kg} / \mathrm{min}, \mathrm{V}_{\mathrm{T}}$ trials were set at 4 , 6 , and $8 \mathrm{~mL} / \mathrm{kg}$, with breathing frequencies set at 25,16 , and 12 breaths/min, respectively (Fig. 3). These $V_{T}$ levels and frequencies were based on the ARDSnet lung-protective ventilator management strategy of lowering $\mathrm{V}_{\mathrm{T}}$ and increasing frequency to maintain a desired minute volume and plateau pressures $<30 \mathrm{~cm} \mathrm{H}_{2} \mathrm{O}$. The following settings remained consistent across all $\mathrm{V}_{\mathrm{T}}$ and breathing frequency trials: $\mathrm{F}_{\mathrm{IO}_{2}}$ of 0.60 , maximum inspiratory flow of $50 \mathrm{~L} / \mathrm{min}$ in a decelerating flow pattern, sensitivity of $3.0 \mathrm{~L} / \mathrm{min}$ (bias flow of $4.5 \mathrm{~L} / \mathrm{min}$ ), and PEEP of $5 \mathrm{~cm} \mathrm{H}_{2} \mathrm{O}$.

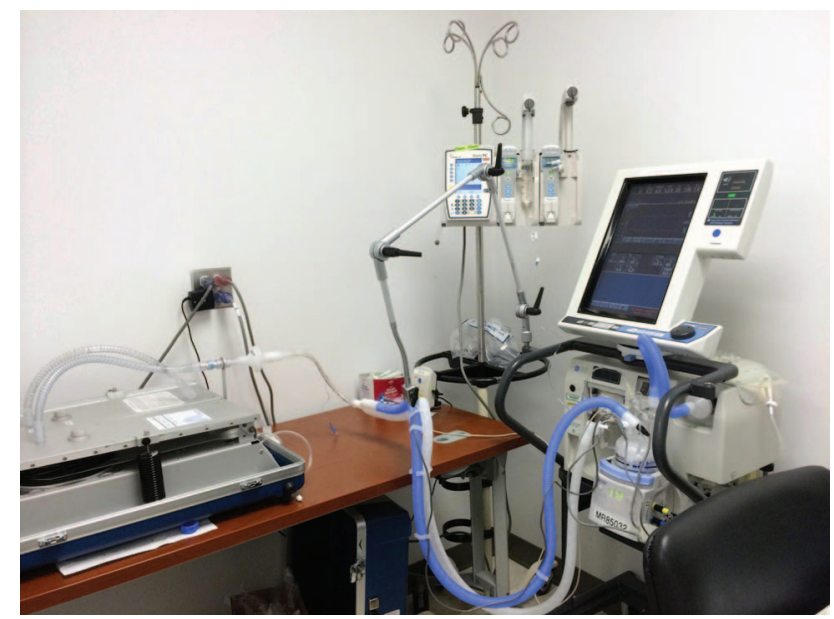

Fig. 2. Full experimental setup.

\section{Nebulizer}

An Aeroneb Solo vibrating mesh nebulizer (Aerogen, Galway, Ireland) was tested according to the manufacturer's instructions to determine its output and was found to meet or exceed the maximum drip rate for drug instillation into the nebulizer's reservoir, with a mean output of $20.184 \mathrm{~mL} / \mathrm{h}$.

\section{Dosing}

Veletri (epoprostenol) was reconstituted with sterile water, according to the manufacturer's instructions, for a concentration of $15,000 \mathrm{ng} / \mathrm{mL}$. The solution was drawn into a 60-mL syringe. A 104-cm (41-inch) segment of intravenous tubing, with a capacity of $6 \mathrm{~mL}$ (ALARIS Medical Systems, San Diego, California) was attached to the syringe and primed once a 20 -gauge needle was attached to the distal end of the intravenous tubing (Fig. 4). The intravenous tubing was clamped to prevent any loss of drug and the potential for overfilling the reservoir before starting nebulization. The needle was used to puncture the silicone plug nebulizer cap and deliver the epoprostenol solution to the Aeroneb Solo reservoir (Fig. 5). The syringe was placed in an Alaris syringe module (Carefusion) (Fig. 6) and programmed to deliver continuous nebulization of epoprostenol at 30, 50, and $70 \mathrm{ng} / \mathrm{kg} / \mathrm{min} .{ }^{4}$ A preliminary run of $20 \mathrm{~min}$, the full duration of an experimental run, was conducted to allow the circuit to stabilize before beginning data collection at each dosage. Each permutation of $\mathrm{V}_{\mathrm{T}}$ and breathing frequency was run for $20 \mathrm{~min}$ and repeated 5 times, for a total of 45 runs with the nebulizer placed at each of the 4 positions. This yielded 180 runs for the entire study. Between each run, the circuit was allowed to stabilize for $1 \mathrm{~min}$. 


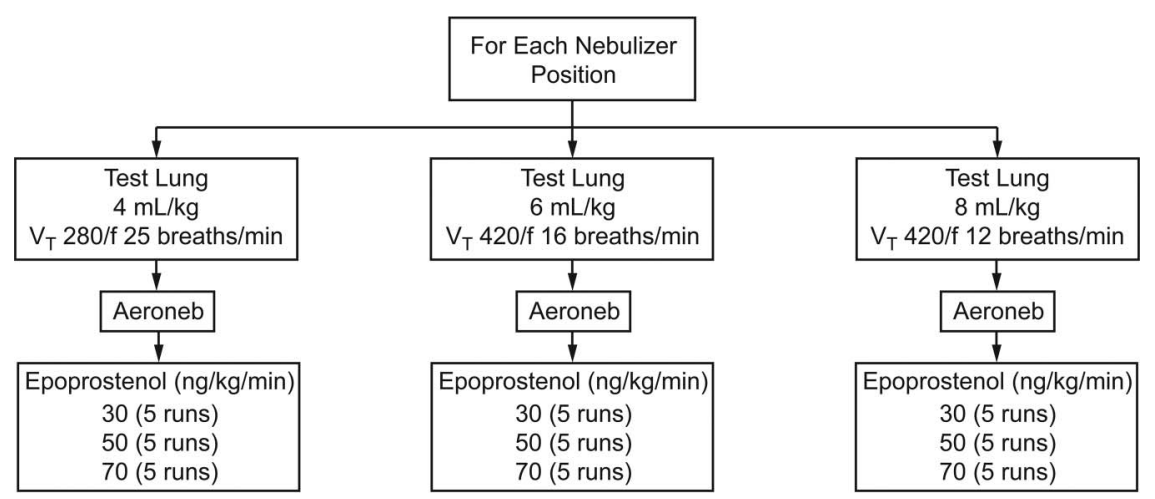

Fig. 3. Schematic of tidal volume and dosage for nebulizer positions. For each position, the 45 measurements shown were conducted.

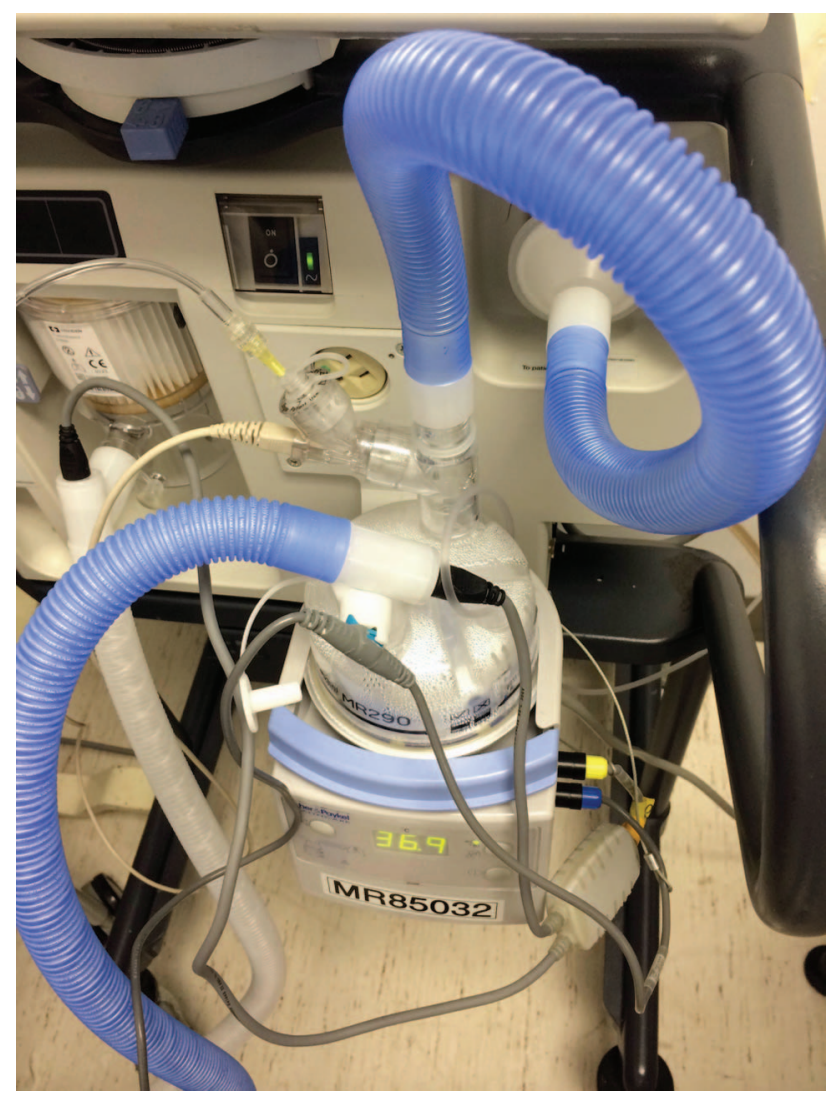

Fig. 4. Vibrating mesh nebulizer placement at the humidifier inlet (dry side).

\section{Laboratory Analysis}

Following each trial, the collecting filter was removed from the circuit. Epoprostenol was eluted from each filter with $10 \mathrm{~mL}$ of sterile water, which was transferred into sterile test tubes. Each sample was analyzed at the institution's core laboratory, which is annually recertified to meet current Centers for Disease Control and Prevention and National Institutes of Health requirements. Samples were analyzed under ultraviolet-visible spectrophotometry at $205 \mathrm{~nm}$ to detect the amount of epoprostenol that had accumulated on the filter.

\section{Data Analysis}

Analysis of data was performed using SPSS 19.0 (SPSS, Chicago, Illinois). Significance was established at $P<.05$. Values of epoprostenol deposition were analyzed using a Kruskal-Wallis test, because the data lacked a normal distribution. If a significant difference was found, post hoc analysis was performed using a Mann-Whitney U test with a Bonferroni correction for multiple comparisons.

\section{Results}

A total of 180 trial runs were conducted in this study, 45 runs at each of 4 nebulizer positions. No runs were excluded from data analysis, because the percentage difference of SD for each group of 5 trial runs was determined to be $<25 \%$.

The mean and SD were calculated for the amount of drug collected for each dose of epoprostenol (no. $=60$ ) (Table 1). A Kruskal-Wallis test showed statistically significant differences across all 3 dosages tested $\left(\chi^{2}=38.8\right.$, $P<.001)$. Pairwise comparisons were made comparing each dosage group with Mann-Whitney tests. Significant differences $(P=.02)$ were found as the dosage was increased. Both the 50-ng/ $/ \mathrm{kg} / \mathrm{min}$ (median $6,065 \mathrm{ng}$, interquartile range [IQR] 3,220-13,002.5 $\mathrm{ng}, P=.001$ ) and 70 $\mathrm{ng} / \mathrm{kg} / \mathrm{min}$ (median 9,890 ng, IQR 6,270-16,140 ng, $P<.001)$ doses were significantly higher than the 30-ng/kg/min dose (median 4,520 ng, IQR 2,285.0$6,712.2 \mathrm{ng})$. The $70-\mathrm{ng} / \mathrm{kg} / \mathrm{min}$ dose was also significantly higher $(P=.006)$ than the $50-\mathrm{ng} / \mathrm{kg} / \mathrm{min}$ dose. The medians and IQRs were calculated for the amount of epoprostenol collected at each of 3 ventilator settings in which $\mathrm{V}_{\mathrm{T}}$ and breathing frequency were varied to maintain a minute volume of $100 \mathrm{~mL} / \mathrm{kg} / \mathrm{min}($ no. $=60$ ). The 


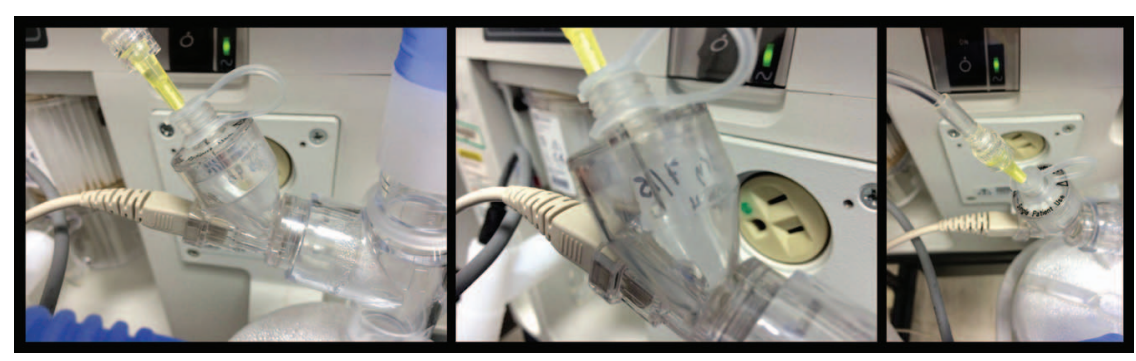

Fig. 5. Vibrating mesh nebulizer and 20-gauge needle conduit.

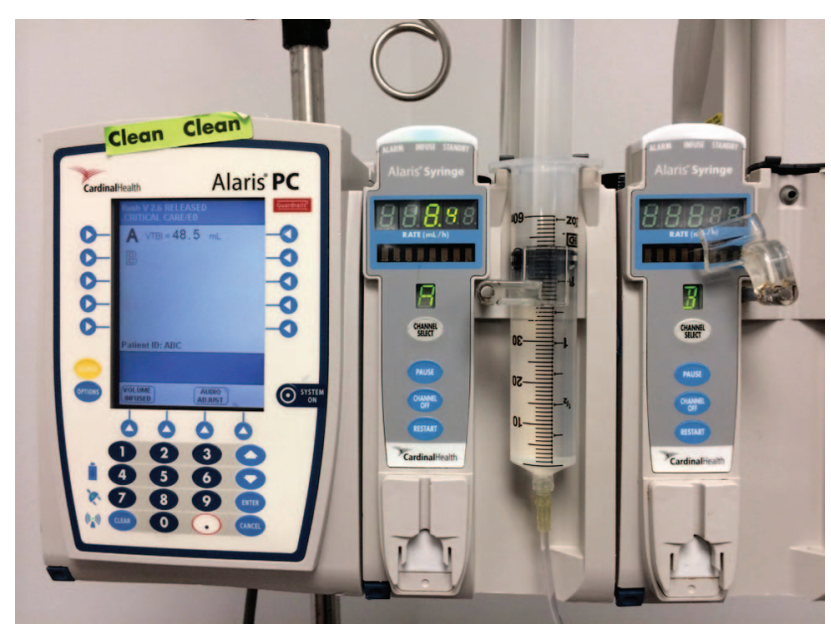

Fig. 6. Intravenous pump with $60-\mathrm{mL}$ syringe.

Table 1. Epoprostenol Dosage and Corresponding Infusion Rate

\begin{tabular}{lccc}
\hline $\begin{array}{c}\text { Dose, } \\
\text { ng/kg/min }\end{array}$ & $\begin{array}{c}\text { Infusion } \\
\text { Rate, mL/h }\end{array}$ & $\begin{array}{c}\text { Total Epoprostenol } \\
\text { Dose, ng/min }\end{array}$ & $\begin{array}{c}\text { Total Epoprostenol } \\
\text { Dose, ng/h }\end{array}$ \\
\hline 30 & 8.4 & 2,100 & 126,000 \\
50 & 14.0 & 3,500 & 210,000 \\
70 & 19.6 & 4,900 & 294,000 \\
\hline
\end{tabular}

Kruskall-Wallis test indicated no significant difference between variations in ventilator settings.

A Kruskal-Wallis test showed statistically significant differences across all 3 dosages tested $\left(\chi^{2}=110.4\right.$, $P<.001)$. Pairwise comparisons were made comparing each of 4 nebulizer positions with each other with MannWhitney tests. Significant differences in deposition were found between nebulizer placement if $P<.008$. Nebulizer placement at the humidifier inlet (median 12,450 ng, IQR 6,755-15,925 ng) or outlet (median 10,050 ng, IQR 7,425$17,710 \mathrm{ng})$ resulted in significantly more drug delivery than when placed at the ETT and Y-piece (median 4,640 ng, IQR 3,850-6,710 ng, $P<.001$ ) or within the inspiratory limb of the circuit (median 2,520 ng, IQR 1,680$3,880 \mathrm{ng}, P<.001)$. The nebulizer placement at the ETT and Y-piece resulted in significantly $(P<.001)$ more drug delivery than when placed within the inspira- tory limb of the circuit. There was not a significant difference in epoprostenol deposition between nebulizer placement at the humidifier inlet and the humidifier outlet (Table 2).

\section{Discussion}

Among the 4 nebulizer positions, the greatest quantity of drug was found to be deposited with the nebulizer placed at the humidifier inlet or outlet. Although there was no significant difference found between those 2 positions, deposition with nebulizer placement at the humidifier inlet was found to be more consistent.

\section{Drug and Nebulizer Selection}

Currently, there is not a delivery system specifically designed for the nebulization of epoprostenol., ${ }^{2,9}$ The MiniHEART Lo-Flo nebulizer (Westmed, Tucson, Arizona) and the Aeroneb Solo (Aerogen) have both been studied ${ }^{2,9}$ in delivering Flolan (GlaxoSmithKline, Research Triangle Park, North Carolina), epoprostenol sodium with a glycine diluent. ${ }^{2}$ The viscosity of the glycine diluent carries the risk of causing ventilator valves to stick. ${ }^{2}$ Veletri (Actelon, San Francisco, California), epoprostenol sodium combined with arginine, mannitol, and sodium hydroxide, ${ }^{2}$ was selected for this study to avoid the potential for drug-related ventilator valve malfunction and for its light and temperature stability. The Aeroneb Solo vibrating mesh nebulizer was selected for this study due to its ability to aerosolize approximately $90 \%$ of the dose placed in the reservoir. ${ }^{10}$ In comparison, jet nebulizers may release only half of the dose. ${ }^{10}$

In a study conducted by Gowda et al, the performance of the Aerogen Solo vibrating mesh nebulizer was found to vary considerably, with nebulization erratically ceasing in $25 \%$ of 55 runs, leaving excessive residual volume within the device's reservoir. ${ }^{11}$ We did not notice the same random cessation in this study, consisting of 180 runs. One nebulizer stopped functioning early in the course of use, was immediately removed from the study, and was replaced with a new device. We did not attempt to restart the 
Table 2. Predicted Epoprostenol Deposition Compared With Actual Mean Drug Collected at the Filter

\begin{tabular}{|c|c|c|c|c|c|c|c|}
\hline \multirow{2}{*}{$\begin{array}{c}\text { Dose, } \\
\mathrm{ng} / \mathrm{kg} / \mathrm{min}\end{array}$} & \multirow{2}{*}{$\begin{array}{l}\text { Infusion } \\
\text { Rate, } \mathrm{mL} / \mathrm{h}\end{array}$} & \multirow{2}{*}{$\begin{array}{c}\text { Total Dose, } \\
\text { ng/min }\end{array}$} & \multirow{2}{*}{$\begin{array}{c}\text { Expected Total } \\
\text { Dose, } \mathrm{ng} / 20 \mathrm{~min}\end{array}$} & \multicolumn{4}{|c|}{ Mean Total Dose, ng \pm SD ( $\%$ delivered $)$} \\
\hline & & & & Dry Side & Wet Side & $\begin{array}{l}\text { Between ETT } \\
\text { and Y-Piece }\end{array}$ & Inspiratory Limb \\
\hline 30 & 8.4 & 2,100 & 42,000 & $6,347 \pm 973(15.1)^{*} \dagger$ & $6,537 \pm 2,234(15.6)^{* \dagger}$ & $3,743 \pm 1,353(8.9) \dagger$ & $1,531 \pm 587(3.6)$ \\
\hline 50 & 14.0 & 3,500 & 70,000 & $13,398 \pm 2,227(19.1)^{* \dagger}$ & $12,383 \pm 5,117(17.7)^{* \dagger}$ & $4,972 \pm 2,096(7.1)$ & $2,354 \pm 904(3.4)$ \\
\hline 70 & 19.6 & 4,900 & 98,000 & $15,697 \pm 2,134(16.0)^{*} \dagger$ & $16,414 \pm 5,129(16.6)^{* \dagger}$ & $7,698 \pm 1,662(7.9) \dagger$ & $4,459 \pm 1,266(4.6)$ \\
\hline \multicolumn{8}{|c|}{$\begin{array}{l}\text { * Significantly higher than between ETT and Y-piece }(P<.001) \text {. } \\
\dagger \text { Significantly higher than inspiratory limb }(P<.001) . \\
\text { ETT = endotracheal tube }\end{array}$} \\
\hline
\end{tabular}

failed device and cannot comment on whether it would have performed differently if it had restarted. We did measure the maximum ouput of each of the nebulizers used to ensure that it exceeded our maximium drip rate of the epoprostenol solution.

\section{Nebulizer Placement}

According to the manufacturer's instructions for use with a ventilator, the Aeroneb Solo should be placed inline on the inspiratory limb of the ventilator circuit before the patient Y-piece. The nebulizer may also be installed at the dry port (inlet) of the humidifier. ${ }^{12}$ Ari et al ${ }^{10}$ determined that positioning a vibrating mesh nebulizer at the humidifier inlet doubles medication delivery when compared with positioning the nebulizer between the patient Y-piece and the circuit.

With ventilator parameters set to deliver a consistent minute volume of $100 \mathrm{~mL} / \mathrm{kg} / \mathrm{min}$, drug deposition increased as the rate of infusion, and thus epoprostenol dosage, was increased (Fig. 7). This direct relationship was seen regardless of nebulizer positioning, with no statistically significant difference in mean drug deposition found between nebulizer placement at the humidifier inlet or outlet. However, these positions resulted in significantly higher drug deposition when compared with nebulizer placement within the inspiratory limb of the circuit or between the endotracheal tube and Y-piece (Fig. 7). This finding is consistent with those of Ari et al, ${ }^{10}$ who determined that the deposition of albuterol was significantly higher when the vibrating mesh nebulizer was placed at the humidifier inlet, with $2 \mathrm{~L} / \mathrm{min}$ of bias flow, instead of between the patient Y-piece and ventilator circuit. A reduction in deposition was seen when bias flow was increased to $5 \mathrm{~L},{ }^{10}$ although in our study, the bias flow was $4.5 \mathrm{~L} / \mathrm{min}$ and the highest deposition was still found with the nebulizer placed at the humidifier inlet.

In a prior study conducted by Ari et al, ${ }^{13}$ the deposition from various nebulizers was evaluated at 3 different locations along the inspiratory limb of the ventilator circuit.

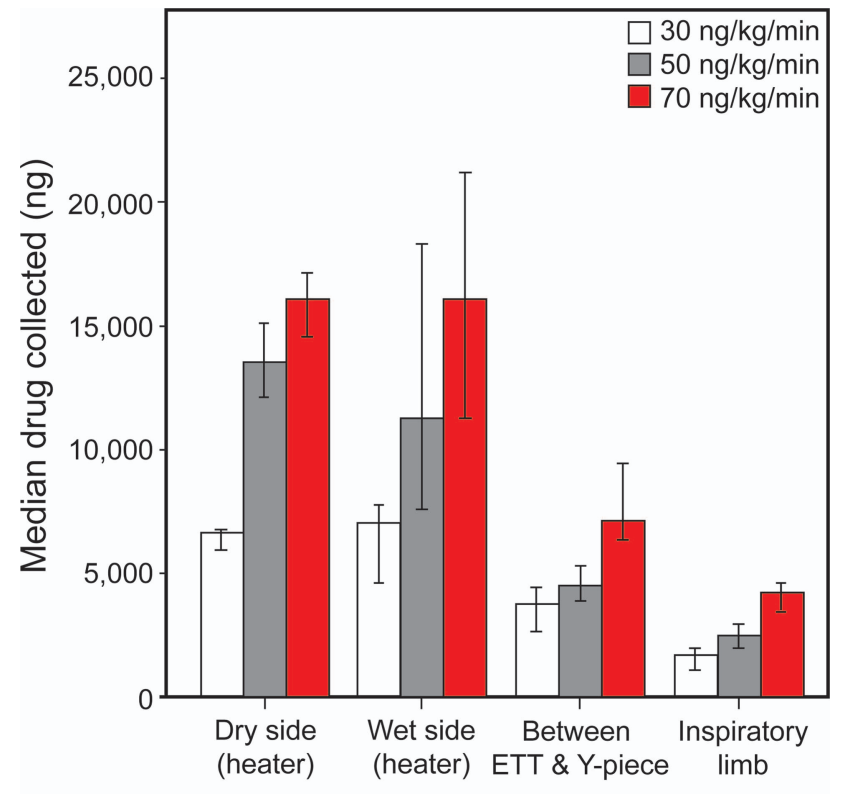

Nebulizer location on heater

Fig. 7. Median epoprostenol collected at each dosage with a comparison of nebulizer placement. Error bars show 95\% Cl. ETT = endotracheal tube.

Position 1 was defined as between the ETT and patient Y-piece; position 2 was defined as $15 \mathrm{~cm}$ from the patient Y-piece; and position 3 was defined as $15 \mathrm{~cm}$ from the ventilator, before the humidifier. ${ }^{13}$ This study did not evaluate a continuous nebulization of medication (albuterol sulfate, $2.5 \mathrm{mg}$ in $3 \mathrm{~mL}$ of normal saline). ${ }^{13}$ In trials assessing the vibrating mesh nebulizer, it was determined that nebulizer placement $15 \mathrm{~cm}$ from the patient $\mathrm{Y}$-piece yielded the greatest deposition of albuterol, followed by placement between the ETT and patient Y-piece; positioning the nebulizer $15 \mathrm{~cm}$ from the ventilator, before the humidifier inlet, yielded the least amount of albuterol deposition. ${ }^{13,14}$ Recommendations from Ari and Fink ${ }^{14}$ for vibrating mesh nebulizer placement suggest that the greatest efficiency for bronchodilator delivery is seen when the nebulizer is placed six inches $(15 \mathrm{~cm})$ from the patient 


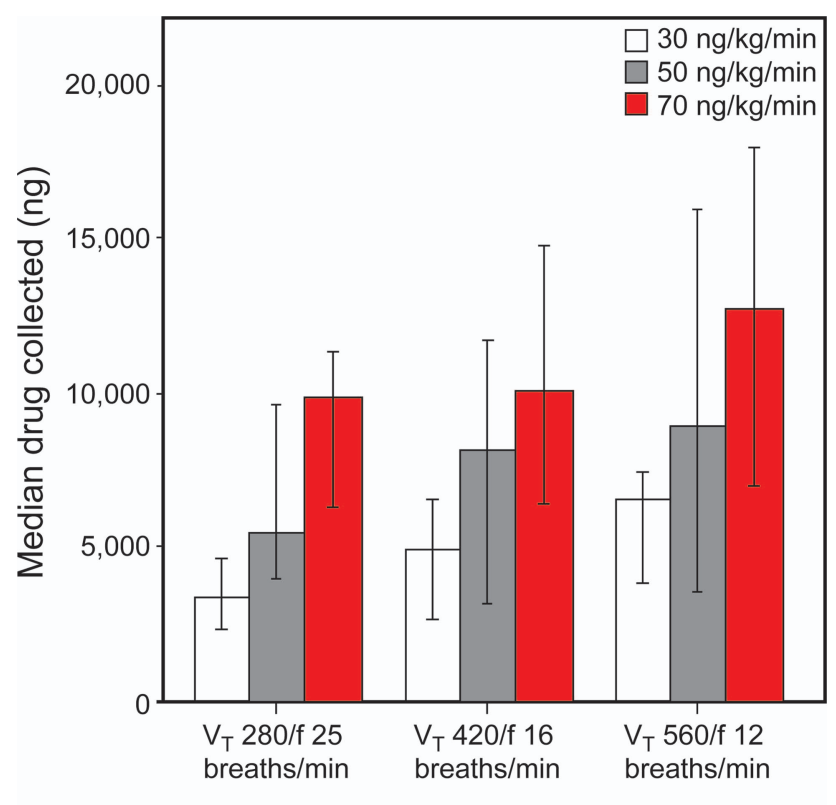

Ventilator setting

Fig. 8. Median epoprostenol collected with changes in ventilator settings. Error bars show $95 \% \mathrm{Cl}$. $\mathrm{V}_{\mathrm{T}}=$ tidal volume $(\mathrm{mL})$.

Y-piece. Inconsistencies between our study and previous research could be a result of differences between ventilators with and without bias flow and/or aerosol particle differences between albuterol and epoprostenol.

A consensus is not yet apparent as to which position provides the most favorable drug deposition in a laboratory setting in studies that evaluate nebulizer placement. Additionally, the ideal position of a nebulizer could potentially change, dependent on the drug being aerosolized. Further study in this area is needed and should include a range of medications to determine whether standard nebulization recommendations should be drug-specific. The clinical importance of variations in drug deposition with nebulizer positioning is also not known. ${ }^{15}$ In a study conducted by Moraine et al, ${ }^{16}$ an ultrasonic nebulizer was positioned before the humidifier in mechanically ventilated subjects (group 1) following open-heart surgery. For subjects randomized to group 2, the nebulizer was placed before the patient Y-piece. Ipratropium bromide was administered, and the amount of drug excreted in each subject's urine was measured through mass spectrometry. No significant difference in the amount of excreted ipratropium bromide was found between the groups. ${ }^{15,16}$

For trial runs conducted with the nebulizer placed at the humidifier outlet, the amount of drug deposited increased as $\mathrm{V}_{\mathrm{T}}$ was increased (Fig. 8). As minute volume was held constant, this increase in deposition is most likely due to increased inspiratory time resulting from the larger administrated $\mathrm{V}_{\mathrm{T}}$ values. ${ }^{14}$ Nebulizer placement at the humidifier inlet did not show this same pattern. At this position, the median drug collected remained consistent despite variations in $\mathrm{V}_{\mathrm{T}}$. Clinically, this stable delivery pattern could reduce the need for titration of continuously nebulized drugs with changes in ventilator settings. A stable delivery pattern could also be of clinical importance when medications with narrow therapeutic indices are trialed for nebulization.

\section{System Stabilization}

When a new vial of epoprostenol was reconstituted and drawn into the infusion syringe, the nebulizer was turned on for a 20-min practice run. This was conducted to allow the circuit to stabilize. The goal of this stabilization period was to begin data collection at a stage approximating continuous nebulization. Although the practice runs were not included in the data analysis, the epoprostenol concentrations eluted from the practice run filters suggest that 20 $40 \mathrm{~min}$ of nebulization is required before consistent amounts of epoprostenol reach the collecting filter at the distal end of the endotracheal tube. This effect was seen to a lesser degree as the infusion rate of the drug was increased. This may be an important consideration when administering aerosolized epoprostenol in a critical care setting.

\section{Limitations}

A limitation of this study is the participation of 3 investigators in data collection but each with a different specific task. One investigator conducted all trial runs with the nebulizer positioned at the humidifier inlet, and another conducted all trial runs with the nebulizer positioned at the humidifier outlet. The final investigator conducted all trial runs with the nebulizer positioned within the inspiratory limb of the circuit and between the endotracheal tube and the Y-piece. Although the individuals trained together and followed the same protocol, slight variations in technique cannot be ruled out. However, this did not cause an apparently negative effect, because the percentage difference of SD for all trial runs was found to be $<25 \%$.

A second limitation may be that of equipment variability. There could be differences in output between the 3 Aeroneb Solo nebulizers used in the study, which may be responsible for alterations in deposition. One nebulizer was dedicated to trial runs conducted with the nebulizer positioned at the humidifier inlet, another was dedicated to trial runs conducted with the nebulizer positioned at the humidifier outlet, and the last nebulizer was dedicated to trial runs conducted with the nebulizer positioned within the inspiratory limb of the circuit and between the endotracheal tube and the Y-piece. The same Aeroneb Solo nebulizers were used for the duration of the study. The frequency at which the nebulizer should be changed when nebulizing epoprostenol is unknown. It was noted that the 
epoprostenol solution left a slightly adhesive residue on containers used in reconstitution. It is possible that this same residue could impact the integrity of the mesh screen over time. For clinical application, accurate drug delivery would probably be dependent on the nebulizer's output being greater than or equal to the drip rate required to manage each patient.

A third limitation is that inconsistencies were apparent in filter absorption. The amount of solution returned following elution was not equal across all trials. In some filters, the sterile water passed easily through the filter medium, whereas very little sterile water passed through other filters. Filters with increased permeability may yield a greater concentration of eluted drug than those that are less permeable. Filters that are less permeable or more absorptive may yield a lesser concentration of eluted drug.

A fourth limitation is the fact that the previous version of the proprietary intravenous adapter for the Aeroneb Solo created a back pressure, resulting in delays in drug instillation instead of a continuous drip rate. To avoid this pattern during the study, a 20-gauge needle was used to puncture the silicone plug cap of the Aeroneb Solo (Fig. 5). Although the needle produced a continuous and consistent drip rate, this setup is not ideal in a patient setting. Since the conclusion of data collection for this study, the intravenous adapter for continuous nebulization with the Aerogen Solo has been modified. As long as the adapter results in a continuous and consistent drip rate, the results would be expected to be similar regrading drug delivery based on placement in the ventilator circuit.

A fifth limitation may be associated with limiting the minute volume at $100 \mathrm{~mL} / \mathrm{kg} / \mathrm{min}$. It would not be unusual to find higher minute volumes in patients with ARDS. This target was chosen because it is a consistent starting point for mechanically ventilated patients at our institution. Keeping this minute volume consistent allowed the impact of lowering $\mathrm{V}_{\mathrm{T}}$ and increasing frequency on drug delivery to be assessed. We cannot draw any conclusions regarding the effect of increasing minute volume in regard to drug delivery in this model.

The study may also be limited by discrepancies between the laboratory and clinical environments. Although each trial run was 20 min in length and was repeated 5 times, this may not be sufficient to reflect continuous nebulization in a clinical setting.

\section{Further Investigation}

It is important to recognize that further work is necessary before these results can be widely accepted. Our study was a first step in this investigation, and additional study is warranted in the utilization of higher minute ventilation, which is often necessary with ARDS patients. Device variability could be addressed by studying multiple nebulizers at each position, and clinical data on subjects are needed to understand the full significance of these findings.

\section{Conclusions}

This study demonstrates the feasibility of the continuous nebulization of epoprostenol solution using a vibrating mesh nebulizer in this bench model. The greatest deposition resulted with nebulizer placement at the humidifier inlet or outlet. Although no significant differences were found, more consistent drug deposition was seen with nebulizer placement at the humidifier inlet. Increasing drip rate to increase the delivered dose to the nebulizer increased mean epoprostenol deposition, as expected. Further clinical study is needed to determine the physiologic and adverse effects of using this system to deliver aerosolized epoprostenol.

\section{ACKNOWLEDGMENTS}

We thank Seby L Edassery for expertise in assay analysis.

\section{REFERENCES}

1. Haj RM, Cinco JE, Mazer CD. Treatment of pulmonary hypertension with selective pulmonary vasodilators. Curr Opin Anaesthesiol 2006; 19(1):88-95.

2. Dzierba AL, Abel EE, Buckley MS, Lat I. A review of inhaled nitric oxide and aerosolized epoprostenol in acute lung injury or acute respiratory distress syndrome. Pharmacotherapy 2014;34(3): 279-290.

3. Afshari A, Brok J, Møller AM, Wetterslev J. Inhaled nitric oxide for acute respiratory distress syndrome and acute lung injury in adults and children: a systematic review with meta-analysis and trial sequential analysis. Anesth Analg 2011;112(6):1411-1421.

4. Fiser SM, Cope JT, Kron IL, Kaza AK, Long SM, Kern JA, et al. Aerosolized prostacyclin (epoprostenol) as an alternative to inhaled nitric oxide for patients with reperfusion injury after lung transplantation. J Thorac Cardiovasc Surg 2001;121(5):981-982.

5. Torbic H, Szumita PM, Anger KE, Nuccio P, LaGambina S, Weinhouse G. Inhaled epoprostenol vs inhaled nitric oxide for refractory hypoxemia in critically ill patients. J Crit Care 2013; 28(5):844-848.

6. Ruan CH, Dixon RA, Willerson JT, Ruan KH. Prostacyclin therapy for pulmonary arterial hypertension. Tex Heart Inst J 2010;37(4): 391-399.

7. Harizi H, Corcuff JB, Gualde N. Arachidonic-acid-derived eicosanoids: roles in biology and immunopathology. Trends Mol Med 2008;14(10):461-469.

8. Ivy DD. Prostacyclin in the intensive care setting. Pediatr Crit Care Med 2010;11(2 Suppl):S41-S45.

9. Dunkley KA, Louzon PR, Lee J, Vu S. Efficacy, safety, and medication errors associated with the use of inhaled epoprostenol for adults with acute respiratory distress syndrome: a pilot study. Ann Pharmacother 2013;47(6):790-796.

10. Ari A, Atalay OT, Harwood R, Sheard MM, Aljamhan EA, Fink JB. Influence of nebulizer type, position, and bias flow on aerosol drug delivery in simulated pediatric and adult lung models during mechanical ventilation. Respir Care 2010;55(7):845-851. 
11. Gowda AA, Cuccia AD, Smaldone GC. Reliability of vibrating mesh technology. Respir Care 2017;62(1):65-69.

12. Aerogen Ltd. Aeroneb Solo System instruction manual. Dangan, Galway, Ireland: Aerogen Ltd.;2014:36.

13. Ari A, Areabi H, Fink JB. Evaluation of aerosol generator devices at 3 locations in humidified and non-humidified circuits during adult mechanical ventilation. Respir Care 2010;55(7):837-844.
14. Ari A, Fink JB. Factors affecting bronchodilator delivery in mechanically ventilated adults. Nurs Crit Care 2010;15(4):192-203.

15. Fink J, Ari A. Aerosol delivery to intubated patients. Expert Opin Drug Deliv 2013;10(8):1077-1093.

16. Moraine JJ, Truflandier K, Vandenbergen N, Berre J, Melot C, Vincent JL. Placement of the nebulizer before the humidifier during mechanical ventilation: effect on aerosol delivery. Heart Lung 2009;38(5):435-439.

This article is approved for Continuing Respiratory Care Education credit. For information and to obtain your CRCE

(free to AARC members) visit 